\title{
All Analog Processing of Speckle affected Self- Mixing Interferometric Signals
}

\author{
Asra A. Siddiqui, Usman Zabit, Member, IEEE, Olivier D. Bernal Member, IEEE, Gulistan Raja, \\ Senior Member, IEEE, and Thierry Bosch, Senior Member, IEEE
}

\begin{abstract}
Self-mixing (SM) or optical feedback interferometry has been extensively used for high precision displacement and vibration sensing. However, presence of speckle can significantly degrade the SM interferometric signal and cause changes in signal amplitude as well as in the operating optical feedback regime, resulting in reduction in measurement precision. Previously, different advanced digital signal processing techniques have been proposed to undo the effects caused by speckle. However, their complex and computationally heavy nature inhibits their use for real-time, high bandwidth sensing applications. In this regard, an all analog signal processing algorithm has been presented in this paper which allows realtime processing of speckle affected SM signal while using standard analog circuits. Various simulations indicated that it is able to correctly process speckle affected SM signals having amplitude variation of at least one order and optical feedback parameter $C$ reduction till 0.5 . This proposed algorithm has been tested on experimentally acquired speckle affected SM signals and found capable of dealing with variations in optical feedback regime and amplitude modulation of SM signals, in accordance with simulation results. The developed hardware prototype circuit measures maximum displacement amplitude of $0.4 \mathrm{~mm}$ at maximum target velocity of $8 \mathrm{~mm} / \mathrm{s}$ for an SM sensor with laser wavelength of $785 \mathrm{~nm}$ as long as $C>0.5$. The proposed all analog processing could be a significant step towards a robust, low-cost, integrated, real-time SM displacement sensor.
\end{abstract}

Index Terms - Interferometry, self-mixing, speckle effect, analog signal processing

\section{INTRODUCTION}

$\mathrm{S}$ ELF mixing interferometry (SMI) or optical feedback interferometry $[1,2]$ is a promising and inherently simple sensing scheme in which the employed laser diode simultaneously acts as a source, detector, as well as microinterferometer. Use of SMI has been demonstrated for imaging [3], distance [4], flow [5], biomedical [6], embedded [7] and mechatronics [8] applications etc.

Displacement and vibration sensing using SMI has been actively researched and many digital signal processing (DSP) algorithms (based on unwrapping of laser phase under optical feedback) have been published [9-13], providing measurement precision ranging from $\lambda / 8$ to $\lambda / 50$. However, in the presence of speckle [14], such high precision SMI displacement retrieval is greatly reduced.

In order to avoid this degradation in performance, two approaches then exist. One approach is to avoid the occurrence of speckle within SMI either by using cooperative target surface, or by adding electro-mechanical/optical/laser [15-17] components to the SMI sensor. The second approach uses additional DSP algorithms for signal recovery such as Hilbert transform [18], envelope extraction [19] and wavelet transform methods $[20,21]$. The later approach is thus preferable as it maintains the inherent simplicity of SMI sensor design. However, significant digital hardware resources are required for real-time implementation of both phase unwrapping and speckle correcting DSP algorithms. This then reduces the scope of SMI sensors for high bandwidth applications e.g. real-time ultrasonic vibration sensing [22].

In this context, the objective of this paper is to present an all analog approach which allows recovery of displacement, even from a speckle affected SMI signal while using no DSP algorithm at all.

All analog implementation of SMI based displacement retrieval has been previously reported in [23]. This method works for moderate regime SM signals having characteristic saw-tooth shaped SMI fringes [24]. The sharp discontinuity within such fringes was used for individual fringe detection while the quasi-linearity of such fringes with remote target motion was used to produce an unwrapped analog output signal. In spite of elegance of this method, presence of speckle drastically degrades the performance of this method (just as it does for digital unwrapping methods [9-13].

The underlying reason is the two-fold impact of speckle on SMI signals: variations in 1) peak to peak amplitude of SMI signal and 2) optical feedback coupling causing a change in SMI operating regime $[1,2]$. Both effects can be observed in an experimentally acquired speckle affected SMI signal shown in Fig. 1. As SMI fringe detection and unwrapping algorithms, including [23], are based on assumption of constant SMI signal amplitude and SMI optical feedback regime, so it is natural that these collapse in case of speckle.

The challenge, in the framework of analog phase unwrapping [23], was as to how speckle affected SMI signal could be processed in an all analog manner. The proposed work shows that the approach of SMI envelope extraction [19], [21] can be implemented in standard analog circuits so that all analog processing of speckle affected SMI signal can be successfully executed. 
this work in other works
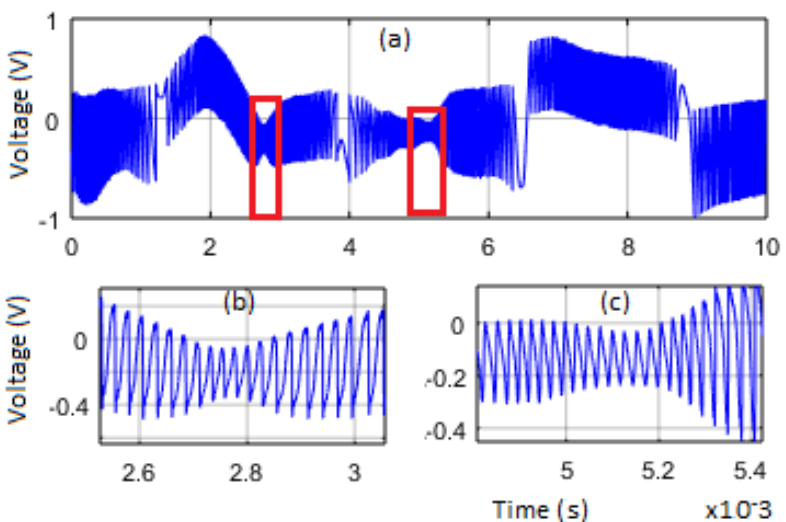

Fig. 1. (a) Exemplar experimental SMI signal affected by speckle. SMI signal segments for displacement (b) towards and (c) away from the sensor. (b) and (c) show changes in amplitude as well as optical feedback regime (from moderate to weak and vice versa) within SMI signal.

Such an all analog processing of speckle affected SMI signal appears full of potential as it enables displacement extraction from SMI signals through standard analog circuits thereby removing constraints such as the requirement of highperformance analog to digital converter (ADC) and DSP hardware platforms. This then not only potentially removes the significant barrier of speckle hindering wide spread use of SMI sensors, but also makes it redundant to employ high performance computing platforms for real-time, high precision, embedded, autonomous SMI sensors.

The rest of the paper is organized as follows: section II presents fundamentals of SMI and speckle, section III summarizes sensor specifications, section IV elaborates the proposed all analog processing, section $\mathrm{V}$ presents results based on experimentally acquired speckle affected SMI signals processed by hardware prototype circuit validating the proposed approach, followed by detailed Discussion about limits of the proposed prototype system and Conclusion.

\section{SELF-MIXING INTERFEROMETRY}

\section{A. SMI Fundamentals}

SMI occurs when a part of laser beam falling on remote target is backscattered and re-enters the active laser cavity thereby interfering with the internal optical field. As a result, variations in laser parameters such as laser wavelength as well as laser output optical power (OOP) occur as a function of variation in optical path length between the laser and the remote target. The variation in OOP, also known as SMI signal, can be expressed as [1]:

$$
P(t)=P_{0}\left[1+m \cdot \cos \left(\varphi_{F}(t)\right)\right]
$$

where $\mathrm{P}_{0}$ represents the emitted optical power under freerunning conditions, $m$ is the modulation index and $\varphi_{F}(t)$ is the laser output phase in the presence of feedback, given by:

$$
\phi_{F}(t)=2 \pi \frac{D(t)}{\lambda_{F}(t) / 2}
$$

where $D(t)$ represents the target displacement.

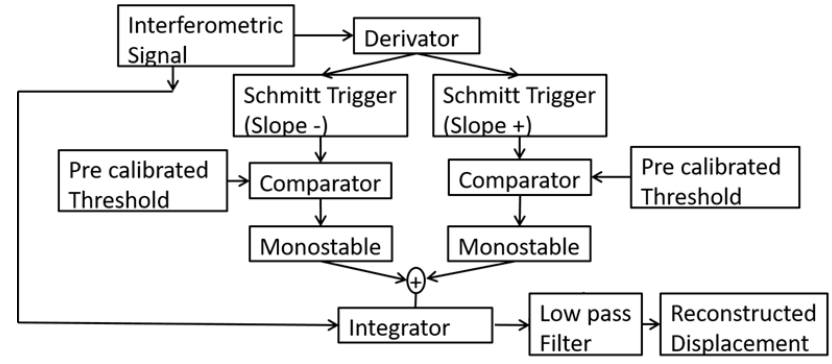

Fig. 2. Schematic block diagram of fully analog SM sensor proposed by Norgia et al. (valid only in the absence of speckle, constant $C$ within moderate feedback regime) achieving SMI fringe detection and SMI phase unwrapping through analog circuits [23].

The emission wavelength subject to feedback $\lambda_{F}(t)$ is given by the excess phase equation [1]:

$$
\phi_{0}(t)-\phi_{F}(t)-C \sin \left[\phi_{F}(t)+\arctan (\alpha)\right]=0
$$

where $\alpha$ is the linewidth enhancement factor, $C$ is the optical feedback coupling factor and $\varphi_{0}(t)$ represents the laser output phase in the absence of feedback, obtained by substituting $\lambda_{F}(t)$ with $\lambda$ in (2), $\lambda$, in this case, is the laser diode emission wavelength under free-running conditions.

$C$ factor plays a fundamental role in SMI and strongly influences the SMI signal characteristics, which can be categorized into the following three major feedback regimes:

1. Weak feedback regime: It corresponds to $0.1<C<1$ in which SM signal fringes have a sinusoidal or asymmetric shape devoid of sharp discontinuity [1].

2. Moderate feedback regime: It relates to $1<\mathrm{C}<4.6$ where SM signal fringes have a saw-tooth shape as well as hysteresis [9].

3. Strong feedback regime: It exists for $\mathrm{C}>4.6$, characterized by a chaotic SM signal [2].

Each fringe in the SMI signal represents a target displacement of $\lambda / 2$. Using simple fringe counting, a sensor would then ensure $\lambda / 2$ resolution by detecting all the fringes. In the absence of speckle, $P(t)$ or SMI signal can be processed by the phase unwrapping methods [9], [12,13] as per (1-3), to provide corresponding $D(t)$ with much better accuracy.

In the absence of speckle, SMI fringes can be easily detected, especially in case of moderate feedback regime [9], [23]. The simplest method applies pre-calibrated constant thresholds on SM derivative signal in order to detect SMI fringes [9], [23], as shown in Fig. 2. Such approach can thus be directly applied to those SMI signals having roughly constant signal amplitude and $1<\mathrm{C}<4$. Such conditions, however, cannot be guaranteed outside laboratory conditions, or in the presence of speckle.

\section{B. Speckle Effect in SM Interferometry}

As speckle pattern induces amplitude modulation to nonlinear frequency modulated SM signal so visually the SM signal appears to have an envelope (see Fig. 1 and Fig. 3) due to its fading as a function of remote target's surface roughness which scatters the incoming laser light resulting in an interference pattern that is granular due to the superposition of 


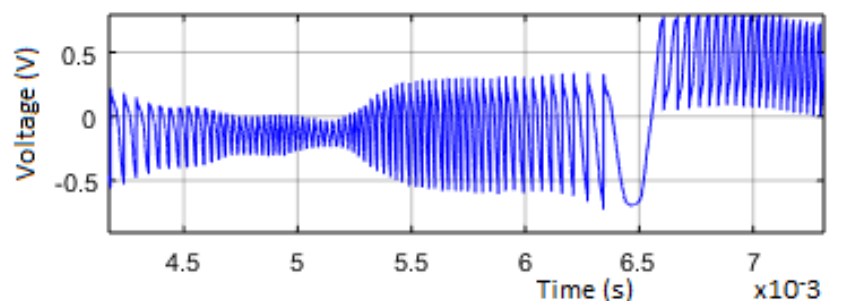

Fig. 3: Deeply modulated segment of exemplar signal affected by Speckle already presented in Fig. 1.

random phases of the beams [21]. For small displacements, it does not affect largely but has a significant effect in the case of large displacements [19]. Let us present a brief literature review regarding speckle in the context of SMI.

Detailed study of error caused by speckle pattern in case of SMI has been conducted in [14] and a solution was presented for phase and amplitude errors generated by speckle pattern in the case of diffusing targets. In addition, work has been carried out in [25] on the statistics of the systematic and random errors in SMI for two cases of developing randomness and fully developed randomness. It was found that both the errors converge to uniform diffuser. This convergence depicts the speckle statistics development.

For high precision SMI displacement recovery, many DSP based algorithms have been proposed to overcome the problems caused by speckle. For example, a Hilbert transform based method calculates the instantaneous phase of the speckle affected SMI signal, which has the interesting property of being unaffected by amplitude modulation of the SMI signal [18]. However, the method suffers from directional ambiguity and uses traditional derivative based threshold approach to ascertain displacement direction [18].

Another DSP algorithm makes use of an envelope extraction technique and thus avoids the use of a constant threshold during the detection of amplitude modulated SMI fringes [19]. It thus employs a dynamic threshold based on local variations in SMI envelopes [19].

Finally, a customized continuous bi-wavelet transform based method has also been proposed in order to robustly detect non-regular SMI fringes [24]. Although it has shown to effectively process speckle affected SMI signals but its utility for real-time, high bandwidth applications remains a concern because of very heavy computational requirements of this algorithm due to use of non-dyadic wavelet decompositions.

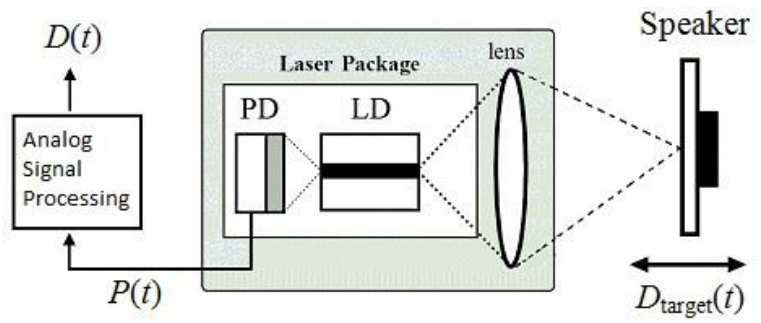

Fig. 4. Schematic block diagram of an analog signal processing based SM sensor setup for displacement sensing of a remote target (loudspeaker).

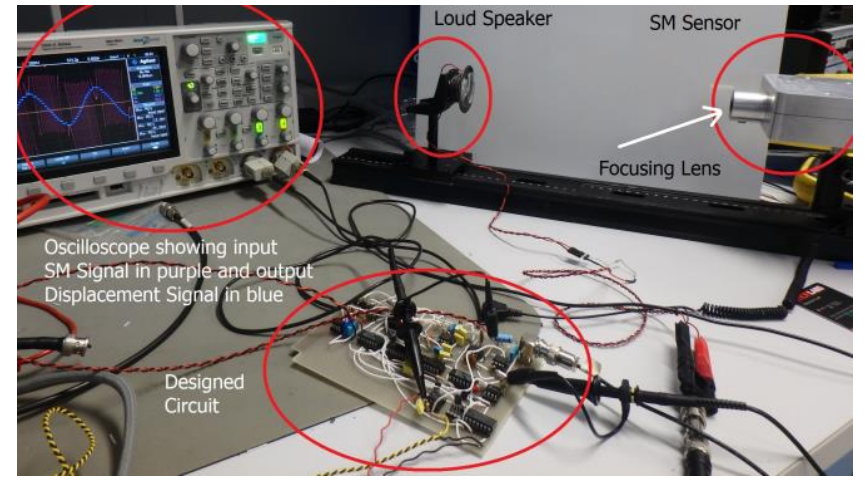

Fig. 5: Photograph of the experimental set-up used for verification of designed hardware circuit for processing of speckle affected SMI signals.

Thus, to summarize, the processing of a speckle affected SMI signal poses significant challenges because speckle causes amplitude modulation of non-linear frequency modulated SM signal (see Fig. 1). The deeply modulated SMI signal has variations in $C$ as well as in peak to peak amplitude. The standard algorithms fail in this scenario due to change from weak feedback regime $(C<1)$ with asymmetrically shaped fringes to moderate feedback regime $(C>1)$ with saw-tooth shaped fringes affected by hysteresis or vice-versa (see Fig. 1). This results in an increase in complexity for detection of SMI fringes. If fringes are not correctly detected then displacement measurement will never be accurate.

\section{Experimental Setup}

The experimental setup, schematized in Fig. 4, was used to acquire experimental SMI signals affected by speckle (photograph of setup is shown in Fig. 5). The deployed SM sensor uses a packaged laser diode (HL7851 by Hitachi ${ }^{\circledR}$ ) with optical output power of $50 \mathrm{~mW}$ at $\lambda=785 \mathrm{~nm}$, operating without current modulation, with a threshold current of 45 $\mathrm{mA}$. It comes with its built-in monitor photodiode (placed at the back facet of the laser diode) which is used to acquire the modulated power signal. A focusing lens was employed to focus the laser beam on the rough metallic surface of the target (a loudspeaker) as seen in Fig. 5.

\section{Proposed Algorithm For Speckle AFFECTED SM SIGNALS}

In this paper, efforts have been exerted to achieve correct SMI fringe detection for a speckle-affected interferometric signal, such as shown in Fig. 3 (which is in fact a deeply modulated portion of exemplar SMI signal of Fig. 1) while using no DSP at all. This signal processing algorithm (summarized by the schematic block diagram shown in Fig. 6) can be implemented in an all-analog manner so as to enable a robust, integrated technique for inherently real-time displacement retrieval from speckle affected SMI signals.

For this purpose, the key aspect is the extraction and subsequent sign-preserving combination of upper and lower envelopes of the amplitude modulated SMI signal. It will be seen that this not only 1) allows the detection of all SMI fringes (whether they are varying in amplitude due to fading or belong to different optical feedback regimes such as weak 


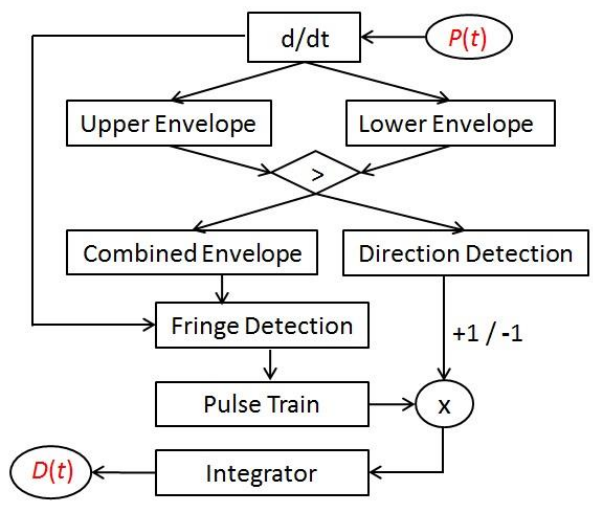

Fig. 6: Schematic block diagram of the proposed all analog signal processing for speckle affected self-mixing interferometric signals.

or moderate feedback regimes) but also 2) preserves the direction of displacement of each fringe.

Thus, the combined envelope serves as an adaptive threshold for fringe detection (by tracking the local peak amplitude of either faded or unaffected SMI fringes) while the sign (positive or negative) of the combined envelope resolves fringe directional ambiguity and direction (forward or backward displacement) can be correctly assigned.

The step by step development of the proposed technique, as per Fig. 6, is presented in the following sub-sections after a short description of the targeted performance specifications.

\section{A. Targeted Performance Specifications}

The proposed targeted analog circuit for displacement retrieval using the previously described SM sensor with $\lambda=$ $785 \mathrm{~nm}$ has the following sensing specifications. The resolution is arbitrarily set to $20 \mathrm{mV} /$ fringe (or $40 \mathrm{mV} / \lambda$ ) as explained in the last subsection. It can be easily tuned by changing the system integrating constant $\tau_{\text {integ. }}$. It is targeted to measure maximum peak to peak displacement amplitude of one thousand fringes $(\approx 0.4 \mathrm{~mm})$ while maximum measurable target velocity is $20 \mathrm{~mm} / \mathrm{s}$. This leads to $\mathrm{T}_{\text {min_fringe }}=20 \mu \mathrm{s}$ approximately which becomes the minimum time period between two consecutive SM fringes. This minimum value has been chosen to be compatible with the employed NE555 monostable, as explained in a later sub-section.

\section{B. Derivative Extraction}

First step of this signal processing is to find the derivative of speckle affected SMI signal (see Fig. 7). To accurately detect each SM fringe, the differentiator circuit is used as it is sensitive to high frequency components of the SM fringes. Here, it has been implemented using an active high-pass filter characterized by a cut-off frequency $\mathrm{f}_{\mathrm{c}}=1 / 2 \pi \tau_{\text {diff. }}$. Note that the amplifier gain bandwidth product (GBW) also directly affects the circuit performance as it filters out the signal frequency higher than GBW. In addition, $f_{c}$ should also be chosen carefully as a high cut-off makes the circuit sensitive to sharp

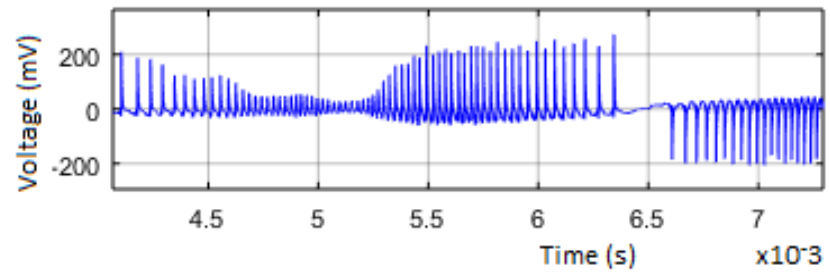

Fig. 7: Simulated derivative extraction of deeply modulated segment of exemplar signal.

discontinuities only while lower values make it sensitive also to $\mathrm{SM}$ signal with lower $C$ values. Nevertheless, a low $\mathrm{f}_{\mathrm{c}}$ will be less effective in removing the low frequency content of SM signal which can affect the envelope tracking system as will be shown later. As a result, a trade-off was chosen here by using low cost amplifiers (MCP6002 by Microchip ${ }^{\circledR}$ with $\mathrm{GBW}=1 \mathrm{MHz})$ and $\tau_{\text {diff }}=0.5 \mathrm{nF} \times 10 \mathrm{k} \Omega$.

\section{Envelope Extraction for Fringe Detection and Direction Recovery}

Next step involves extraction of envelopes of the SM derivative signal. As previously stated, this is the fundamental step in the proposed technique. In order to correctly track the local peak amplitude of various SMI fringes (whether they belong to any specific regime or exhibit fading) making up the SMI signal, two envelopes are extracted in an analog manner. One is named as positive envelope and other is named as negative envelope (see Fig. 8).

The used envelope extraction of derivative utilizes an AM (amplitude modulation) demodulator. For demodulation, an asynchronous detector is required as the signal is affected by speckle (as a function of target surface roughness) and hence information about the modulating frequency is not available. For this purpose, a precision rectifier based on a diode is implemented (see Fig. 9).

So, the differentiator output is fed into this stage as input. In the first step of envelope detection, half-wave rectification is carried out to obtain a positive half of the signal. After rectification, a low pass filter (bigger blue circle in Fig. 9) and a memory loss circuit (smaller orange circle in Fig. 9) are implemented to perform envelope extraction.

As shown in Fig. 8, the envelopes are not only 1) required to make the scheme less sensitive to speckle and to noise for the sake of fringe detection by following the amplitude
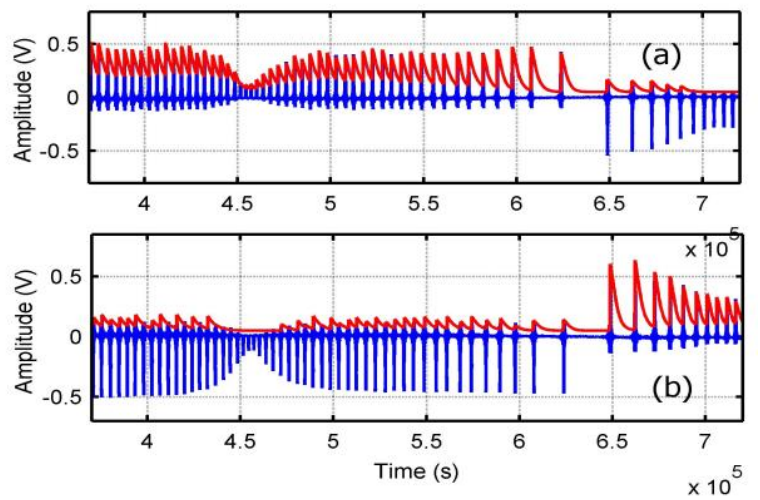

Fig. 8: (a) Simulated derivative of speckle affected SM signal (in blue) and positive envelope (in red), (b) inverted derivative (in blue) and negative envelope (in red). 


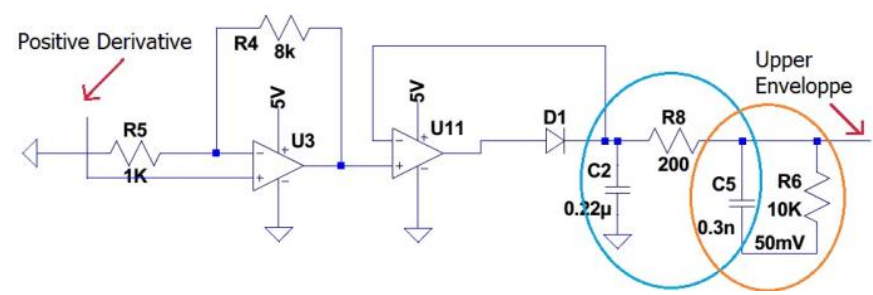

Fig. 9: Positive envelope detector designed using op amps and diode.
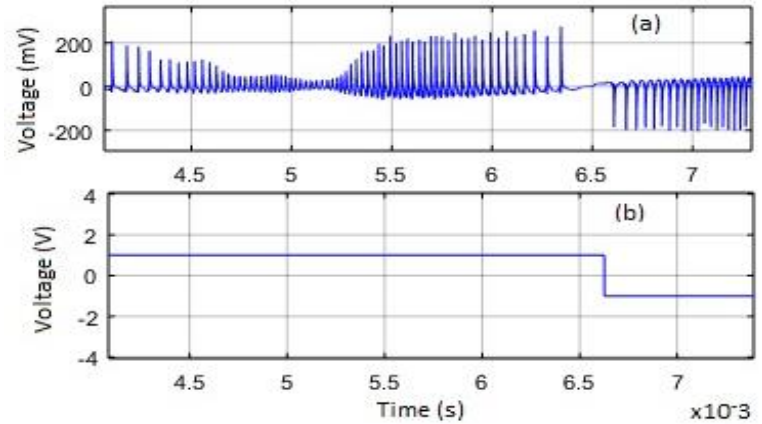

Fig. 10: (a) Simulated derivative of deeply modulated region of Exemplar signal, and (b) sign of combined envelope of derivative preserving the direction of fringes.

variation of the SM derivative signal but also to 2) determine the displacement direction obtained by comparing both the envelope amplitudes (as shown later in Fig. 10). Consequently, a trade-off is necessary to set the time-constant of envelope detector $\tau_{\text {envelope }}$ since a large value $\tau_{\text {envelope }}$ allows a better immunity to noise but a poor tracking ability regarding displacement direction change and speckle affected $\mathrm{SM}$ signal amplitude. Here, $\tau_{\text {envelope }}=\mathrm{R}_{\mathrm{eq}} \mathrm{C}_{\mathrm{eq}}=2.2 \mathrm{~ms}$ was chosen. Similarly, a negative envelope of derivative is tracked, as shown in Fig. 8.

\section{SMI Fringe Detection and Pulse Generation}

Final step of this signal processing is to localize or detect the SMI fringes affected by speckle. Two comparators are employed for this purpose (one for the positive and one for the negative SM envelope). The two inputs of comparator are the amplified derivative of $V_{S M}$ (original speckle affected SM signal) and positive or negative $\mathrm{V}_{\text {ENVELOPE }}$ (envelope of derivative of SM signal). So, If the derivative is greater than the envelope then the comparator switches to a high voltage (indicative of presence of fringe), otherwise its output stays at ground level. Fig. 11 (b) presents how this allows correct detection of all fringes, including the deeply modulated SMI fringes.

Once SMI fringes are correctly detected with correct fringe direction, then it necessary to generate a finite constant duration pulse for each detected fringe. Note that the duration of pulses should be exactly the same so as to generate equal up and down steps after integration. As opposed to [27] where
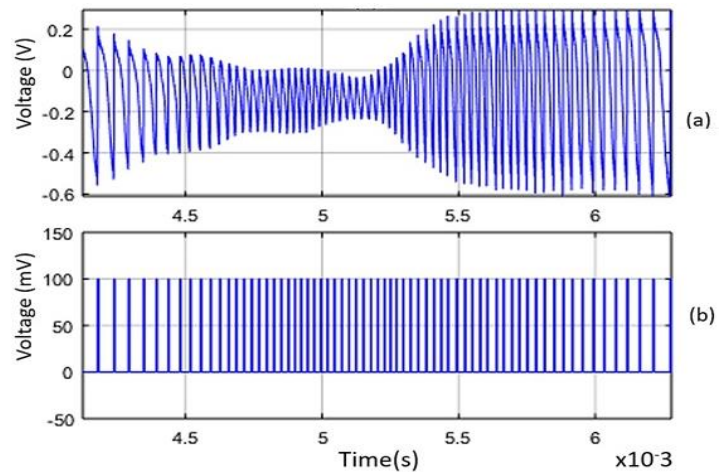

Fig. 11: (a) Simulated SM signal (b) Comparator output showing transitions for the detection of fringes.

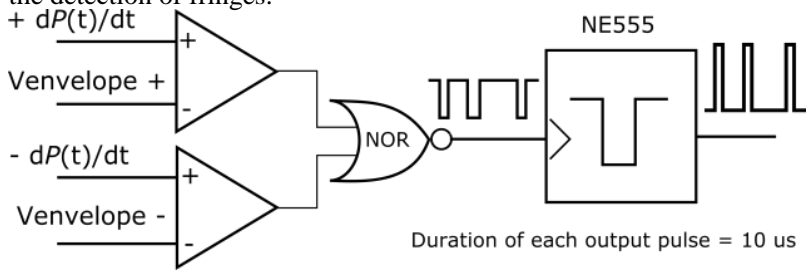

Fig. 12: Schematic diagram of circuit for SM fringe detection and pulse train generation by using a single monstable (NE555) triggered either by the positive or the negative detected fringe.

two monostable circuits were used, here only one monostable circuit (NE555 triggered either by the positive or the negative fringe) is used to generate a unique pulse train (see Fig. 12). The pulse duration is set to the minimum value allowed by the NE555 i.e. $T_{\text {pulse }}=10 \mu$ s. This implies that in the best case two successive fringes can be successfully detected if $\Delta \mathrm{t}>10 \mu \mathrm{s}$.

\section{E. Charge Pump and Integration}

After all the pulses have been generated, it is necessary to distinguish the pulse corresponding to a rising or a falling fringe by using the direction information provided by the result of comparison of envelopes. A circuit called charge pump is thus used here to feed an integrator as shown in Fig 13. The integrator is designed for the pulse accumulation. The integrator thus provides an output signal in the shape of a staircase signal where each step $(\Delta \mathrm{V})$ of the staircase represents $\lambda / 2$ displacement. Hence, it results in a staircase signal that approximates the remote target's displacement with a rough resolution of $\lambda / 2$. The integrating time constant $\tau_{\text {integ }}=$ $\mathrm{C}_{\text {integ }} \mathrm{R}_{\text {integ }}=68 \mathrm{nF} \times 22 \mathrm{k} \Omega \approx 1.5 \mathrm{~ms}$ is set to generate an output $\Delta \mathrm{V} \approx 20 \mathrm{mV}$ per detected fringe.

\section{RESULTS}

\section{A. Design Validation}

The proposed analog signal processing algorithm was initially validated in MATLAB ${ }^{\circledR} 7.0$ Simulink $^{\circledR}$ developed by MathWorks ${ }^{\circledR}$. Afterwards, analog circuit based validation was achieved by using analog circuit simulation software, called LT SPICE IV ${ }^{\circledR}$ by Linear Technology ${ }^{\circledR}$.

\section{B. Processing of Exemplar Signal}

The proposed analog signal processing has been able to correctly detect all fringes contained in the exemplar SMI signal having significant amplitude modulation. Correct detection and direction recovery of these modulated SMI fringes is shown in Fig. 10 and Fig. 11 respectively. 


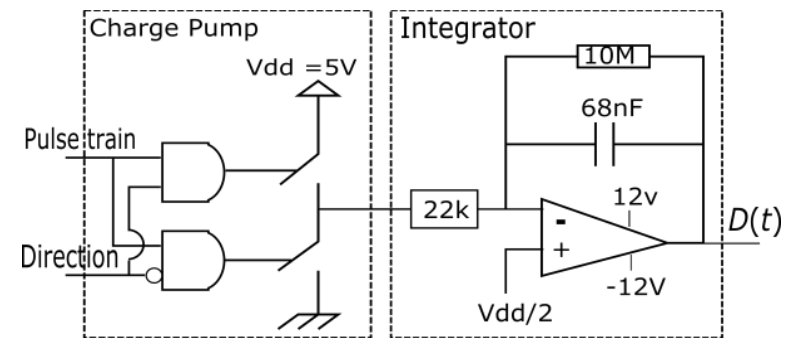

Fig. 13: Charge pump circuit used to feed the integrator circuit.

(a)

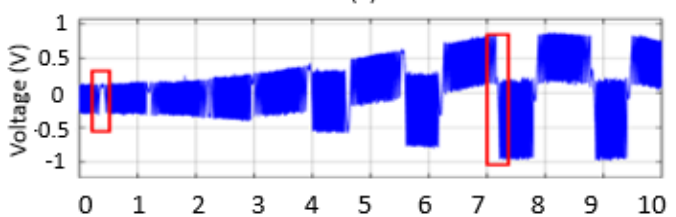

(b) Weak Feedback regime

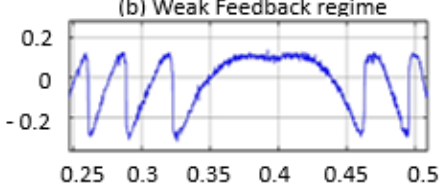

(c) Moderate Feedback regime
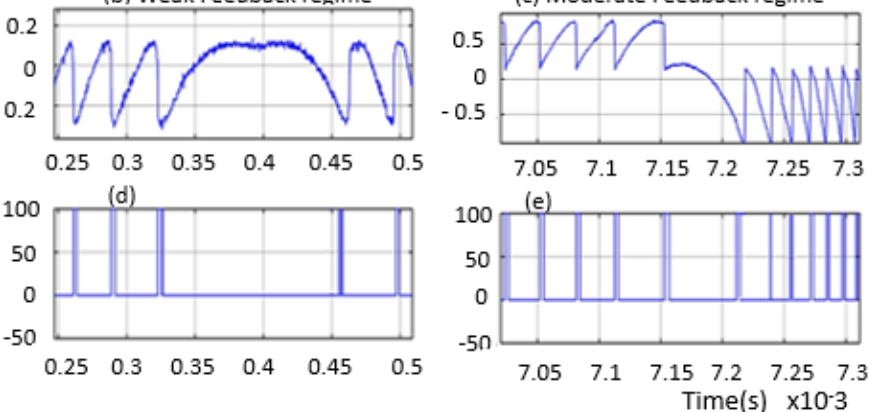

Fig. 14: (a) Experimental speckle affected SMI signal with its (b) weak optical feedback regime segment, (c) moderate optical feedback regime segment, while (d-e) show correct detection of SMI fringes of both regimes.

\section{Moderate Feedback Regime}

For an experimental speckle affected SMI signal, corresponding to harmonic target vibration of $25 \mu \mathrm{m} p$-p amplitude, with moderate feedback regime segments (Fig. 14), the proposed analog signal processing is equally able to process this signal without any change in its parameters. This enables autonomous detection of all moderate feedback regime SMI fringes as shown in Fig. 14 (c) and (e).

\section{Weak Feedback Regime}

The same analog design correctly processes the weak feedback regime segments of the experimentally acquired SMI signal of Fig. 14 as well. The signal with $C<1$ segment can be seen in Fig. 14 (b) while Fig. 14 (d) represents the correct detection of corresponding weak regime SMI fringes.

Such a performance is achieved because the proposed analog processing tracks upper and lower envelopes of the speckle affected signal at all times to detect fringes and makes use of the sign of combined envelope to find the correct fringe direction by comparing upper and lower envelopes at every instant to find which one has larger amplitude. This results in correct fringe localization as well as direction identification.

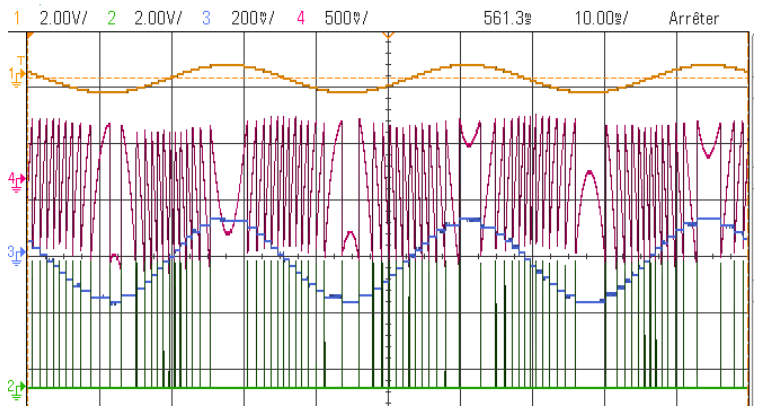

Fig. 15: Screenshot from oscilloscope showing hardware prototype based displacement retrieval in the absence of speckle. From top to bottom: speaker excitation signal in brown (channel 1), experimental SM signal without speckle in purple (channel 4), unwrapped output displacement signal in blue (channel 3), and pulse train indicating detection of each SMI fringe in green (channel 2).

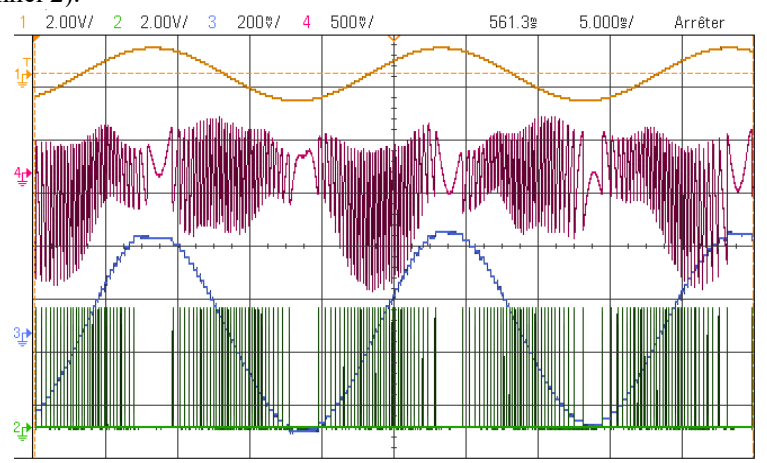

Fig. 16: Screenshot from oscilloscope showing hardware prototype based displacement retrieval in the presence of speckle. From top to bottom: target vibration excitation signal in brown (channel 1), experimental SM signal affected by speckle in purple (channel 4), unwrapped output displacement signal in blue (channel 3), and pulse train indicating detection of each SMI fringe in green (channel 2).

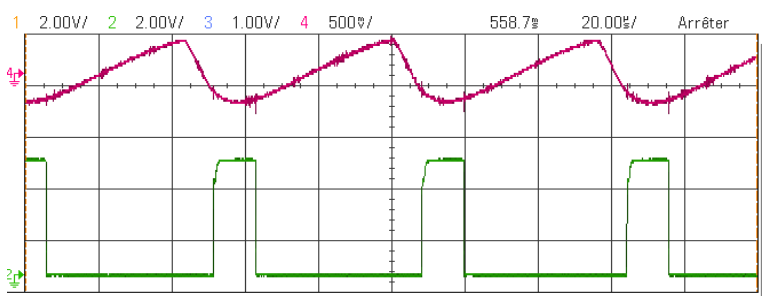

Fig. 17: Oscilloscope screenshot showing the performance of the hardware circuit in terms of maximum measurable target velocity of approximately 8 $\mathrm{mm} / \mathrm{s}$ corresponding to inter-fringe delay of $50 \mu \mathrm{s}$. Pulse duration of $12 \mu \mathrm{s}$ can also be seen for the pulse train signal in green (channel 2).

\section{E. Experimental Hardware Prototype}

The proposed circuit has been implemented in hardware and tested by using real-time experimental SM signals, both in the presence and absence of speckle (see Fig. 5 for set-up).

Fig. 15 and Fig. 16 (corresponding to harmonic target vibration with $5 \mu \mathrm{m}$ and $14 \mu \mathrm{m}$ amplitude respectively) show screenshots from oscilloscope indicating how the prototype circuit has correctly achieved SMI fringe detection in the absence as well as in the presence of speckle respectively.

The performance of the developed prototype has been tested in terms of its ability to correctly process a maximum density of SM fringes (as seen in Fig. 17). The prototype has thus been found to process only up to $\mathrm{T}_{\text {min_fringe }}=50 \mu \mathrm{s}$ (see Fig. 17). This then equates to maximum measurable target velocity 
of approx. $8 \mathrm{~mm} / \mathrm{s}$. A reason for reduced performance of developed proto-type is due to low gain-bandwidth product $(\mathrm{GBW}=1 \mathrm{MHz})$ of employed op-amps $(\mathrm{MCP} 6002$ by Microchip ${ }^{\circledR}$ used as comparator and amplifier). Better performance could thus be obtained by using high performance op-amps.

\section{DISCUSSION}

\section{A. Estimation of Minimum Recoverable SMI Signal}

The performance of the developed prototype in terms of its ability to process SMI signals with varying $C$ value and fringe amplitude was tested by emulating different SMI signals (see Table I). These SMI signals were emulated by using an arbitrary function generator (AFG) by Tektronix $^{\circledR}$, model AFG3000. SMI signals corresponding to $5 \mu \mathrm{m}$ amplitude were loaded into AFG with $0.5<C<4$. Then, peak to peak amplitude of each SMI signal was reduced from $2.5 \mathrm{~V}$ (max. output voltage of $\mathrm{AFG}$, denoted $\mathrm{A}_{\max }$ ) to a lower amplitude. The smallest SMI signal amplitude which still resulted in correct processing was noted as $\mathrm{A}_{\min }$.

Table I

PERFORMANCE OF PROTOTYPE CIRCUIT AS A FUNCTION OF MINIMUM DETECTABLE FRINGE AMPLITUDE AND C FACTOR

\begin{tabular}{|c|c|c|c|}
\hline \hline $\begin{array}{c}C \\
\text { value }\end{array}$ & $\begin{array}{c}\text { Ratio }= \\
A_{\max }(\mathrm{V}) / A_{\min }(\mathrm{V})\end{array}$ & $\begin{array}{c}\text { Correct } \\
\text { Fringe } \\
\text { Detection }\end{array}$ & $\begin{array}{c}\text { Correct } \\
\text { Direction } \\
\text { Detection }\end{array}$ \\
\hline 4 & $2.5 / 0.07=35.7$ & Yes & Yes \\
\hline 3.5 & $2.5 / 0.05=50.0$ & Yes & Yes \\
\hline 3 & $2.5 / 0.045=55.5$ & Yes & Yes \\
\hline 2.5 & $2.5 / 0.03=83.3$ & Yes & Yes \\
\hline 2 & $2.5 / 0.03=83.3$ & Yes & Yes \\
\hline 1.5 & $2.5 / 0.04=62.5$ & Yes & Yes \\
\hline 1 & $2.5 / 0.12=20.8$ & Yes & Yes \\
\hline 0.8 & $2.5 / 0.3=8.3$ & Yes & Yes \\
\hline 0.6 & $2.5 / 0.4=6.2$ & Yes & Yes \\
\hline
\end{tabular}

Expectedly, for $C<2$, the lower the $C$ value, the higher is $\mathrm{A}_{\min }$. Similar trend is seen for $C>2$. Both trends are expected as SMI fringes become increasingly smaller in amplitude for $C$ $>2$ while SMI fringes become increasingly devoid of sharp discontinuity as $C<2$.

An experimental SMI signal, processed by developed prototype circuit, indicating the limit of proposed system, is shown in light blue color in Fig. 18. It exhibits general amplitude modulation whereas a specific portion of it has been significantly affected by the presence of dark speckle, as seen in Fig. 18. This specific portion indicates that the local SM signal amplitude and $\mathrm{C}$ value have been significantly reduced while there is also presence of wide band noise.

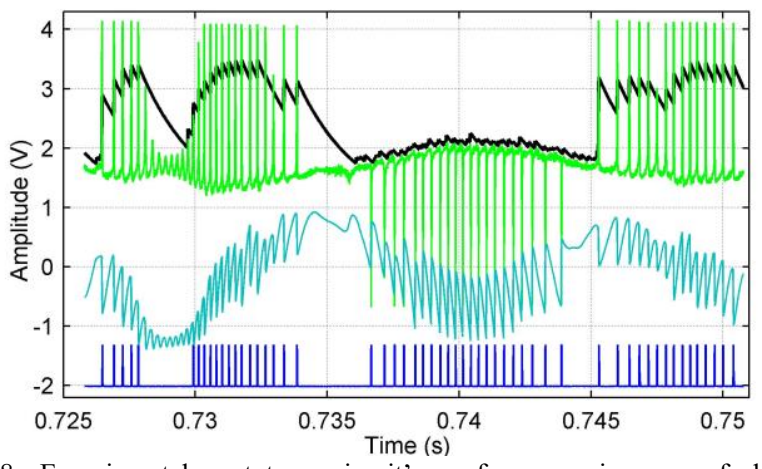

Fig. 18: Experimental prototype circuit's performance in case of deep modulation of SM signals due to occurrence of dark speckle resulting in reduction of local $C$ value as well as signal to noise ratio. It shows inability of the proposed system to detect SM fringes with $C<0.5$. From top to bottom: differentiated SM signal in green, upper envelope in black, speckle affected experimental SM signal in light blue, and pulse train in dark blue.

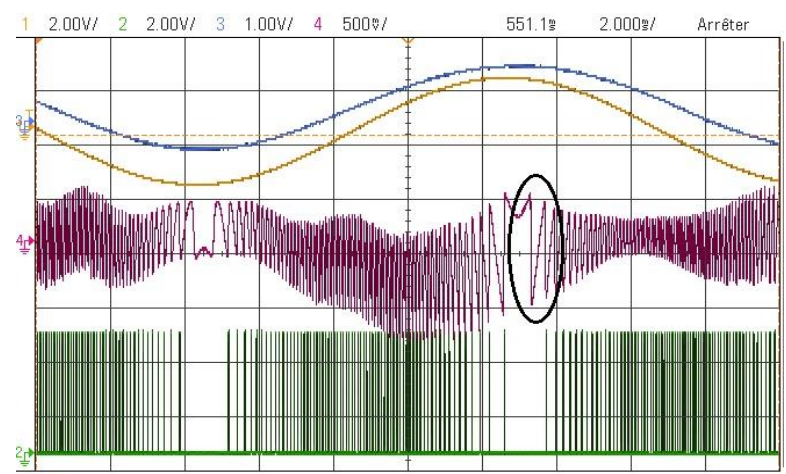

Fig. 19: Screenshot from oscilloscope showing impact of hardware prototype's envelope detector in terms of missed detection of first SM fringe occurring after direction reversal (encircled in black). From top to bottom: unwrapped output displacement signal in blue (channel 3), target vibration excitation signal in brown (channel 1), experimental SM signal affected by speckle in purple (channel 4), and pulse train indicating detection of each SMI fringe in green (channel 2).

\section{B. Impact of Envelope Detector}

In the absence of amplitude modulation of SM fringes, the proposed system can continue to measure target velocity of up to $8 \mathrm{~mm} / \mathrm{s}$. However, in the presence of amplitude modulation of such nature that the amplitude of next differentiated fringe instantaneously becomes half of the previously detected differentiated fringe (as opposed to a low amplitude modulation in which the amplitude of the next differentiated fringe is slightly reduced) then this means that the envelope of the previously detected differentiated fringe would require a time equal to $\Delta \mathrm{T}_{\text {fringe }}=0.7 * \tau_{\text {envelope }}=0.7 * 2.2 \mathrm{~ms}=1.5 \mathrm{~ms}$ in order that its amplitude reduces to half of its peak amplitude. This dead-time of $1.5 \mathrm{~ms}$ imposes that any next fringe with half-amplitude cannot be correctly processed before $1.5 \mathrm{~ms}$. This is clearly shown in prototype based Fig. 18 (green line for the differentiated fringe signal and black line for the tracking envelope). Thus, the maximum measurable velocity reduces to $0.27 \mathrm{~mm} / \mathrm{s}$ under the condition of instantaneous differentiated fringe amplitude reduction by half between two consecutive fringes separated by $\Delta \mathrm{T}_{\text {fringe }}=1.5 \mathrm{~ms}$.

Note also that a large $\tau_{\text {envelope }}$ can also induce a late displacement direction reversal notification to the system. This 
can result in one undetected fringe occurring just after each direction reversal (Fig. 19). This is also a current limitation of the system.

\section{CONCLUSION}

Above mentioned characteristics of proposed signal processing provide a strong justification of an all analog implementation. This proposed signal processing, validated by a prototype circuit tested to measure up to $8 \mathrm{~mm} / \mathrm{s}$ with displacement span of $0.4 \mathrm{~mm}$, is a basic step towards the physical implementation of a robust analog algorithm for speckle affected SM signals. It is also capable of effectively dealing with moderate and weak optical feedback regimes in order to detect the correct location and direction of interferometric fringes present in the SM signal, as long as $\mathrm{C}>$ 0.5. After refinement, it is possible to integrate it in a low-cost opto-electronic sensor that can be used for real-time analog displacement measurement even in the corruptive presence of speckle, thus enlarging the scope of SM sensors for practical real-world sensing applications, where occurrence of speckle often becomes unavoidable.

\section{REFERENCES}

[3] M. Wienold, T. Hagelschuer, N. Rothbart, L. Schrottke, K. Biermann, H. Grahn, et al., "Real-time terahertz imaging through self-mixing in a quantum-cascade laser," Applied Physics Letters, vol. 109, p. 011102, 2016.

[4] M. Norgia, G. Giuliani, and S. Donati, "Absolute distance measurement with improved accuracy using laser diode selfmixing interferometry in a closed loop," IEEE Transactions on Instrumentation and Measurement, vol. 56, pp. 1894-1900, 2007.

[5] M. Norgia, A. Pesatori, and L. Rovati, "Self-mixing laser Doppler spectra of extracorporeal blood flow: a theoretical and experimental study," IEEE Sensors Journal, vol. 12, pp. 552-557, 2012.

[6] S. Donati and M. Norgia, "Self-mixing interferometry for biomedical signals sensing," IEEE Journal of Selected Topics in Quantum Electronics, vol. 20, pp. 104-111, 2014.

[7] U. Zabit, O. D. Bernal, and T. Bosch, "Design and analysis of an embedded accelerometer coupled Self-Mixing laser displacement sensor," IEEE Sensors Journal, vol. 13, pp. 2200-2207, 2013.

[8] S. Ottonelli, M. Dabbicco, F. De Lucia, M. Di Vietro, and G. Scamarcio, "Laser-self-mixing interferometry for Mechatronics Applications," Sensors, vol. 9, pp. 3527-3548, 2009.

[9] C. Bes, G. Plantier, and T. Bosch, "Displacement measurements using a self-mixing laser diode under moderate feedback," IEEE Transactions on Instrumentation and Measurement, vol. 55, pp. 1101-1105, 2006

[10] A. L. Arriaga, F. Bony, and T. Bosch, "Real-time algorithm for versatile displacement sensors based on self-mixing interferometry," IEEE Sensors Journal, vol. 16, pp. 195-202, 2016.

[11] O. D. Bernal, U. Zabit, and T. M. Bosch, "Robust method of stabilization of optical feedback regime by using adaptive optics for a self-mixing micro-interferometer laser displacement sensor," IEEE Journal of Selected Topics in Quantum Electronics, vol. 21, pp. 336-343, 2015.

[12] S. Merlo and S. Donati, "Reconstruction of displacement waveforms with a single-channel laser-diode feedback interferometer," IEEE Journal of Quantum Electronics, vol. 33, pp. 527-531, 1997.
[13]

Y. Fan, Y. Yu, J. Xi, and J. F. Chicharo, "Improving the measurement performance for a self-mixing interferometry-based displacement sensing system," Applied Optics, vol. 50, pp. 50645072, 2011.

[14] S. Donati, G. Martini, and T. Tambosso, "Speckle pattern errors in self-mixing interferometry," IEEE Journal of Quantum Electronics, vol. 49, pp. 798-806, 2013.

[15] M. Norgia and S. Donati, "A displacement-measuring instrument utilizing self-mixing interferometry," IEEE Transactions on Instrumentation and Measurement, vol. 52, pp. 1765-1770, 2003.

[16] M. Norgia, S. Donati, and D. D'Alessandro, "Interferometric measurements of displacement on a diffusing target by a speckle tracking technique," IEEE Journal of Quantum Electronics, vol. 37, pp. 800-806, 2001.

[17] R. Atashkhooei, S. Royo, and F. J. Azcona, "Dealing with speckle effects in self-mixing interferometry measurements," IEEE Sensors Journal, vol. 13, pp. 1641-1647, 2013.

[18] A. L. Arriaga, F. Bony, and T. Bosch, "Speckle-insensitive fringe detection method based on Hilbert transform for self-mixing interferometry," Applied Optics, vol. 53, pp. 6954-6962, 2014.

[19] U. Zabit, O. D. Bernal, and T. Bosch, "Self-mixing laser sensor for large displacements: Signal recovery in the presence of speckle," IEEE Sensors Journal, vol. 13, pp. 824-831, 2013.

[20] O. D. Bernal, H. C. Seat, U. Zabit, F. Surre, and T. Bosch, "Robust Detection of Non-Regular Interferometric Fringes From a SelfMixing Displacement Sensor Using Bi-Wavelet Transform," IEEE Sensors Journal, vol. 16, pp. 7903-7910, 2016.

[21] A. Jha, F. J. Azcona, C. Yañez, and S. Royo, "Extraction of vibration parameters from optical feedback interferometry signals using wavelets," Applied Optics, vol. 54, pp. 10106-10113, 2015.

[22] Y. Tao, M. Wang, and D. Guo, "Compound cavity theory of resonant phase modulation in laser self-mixing ultrasonic vibration measurement," Optical Engineering, vol. 55, pp. 074107-074107, 2016.

[23] M. Norgia and A. Pesatori, "Fully analog self-mixing laser vibrometer," in Instrumentation and Measurement Technology Conference (I2MTC), 2011 IEEE, 2011, pp. 1-4.

[24] O. D. Bernal, U. Zabit, and T. Bosch, "Classification of laser selfmixing interferometric signal under moderate feedback," Applied Optics, vol. 53, pp. 702-708, 2014.

[25] S. Donati and G. Martini, "Systematic and random errors in selfmixing measurements: effect of the developing speckle statistics," Applied Optics, vol. 53, pp. 4873-4880, 2014. 